\title{
Experimental Drift Velocity Field in Transistor Devices
}

\author{
S. SAYGI \\ Department of Physics, Faculty of Art and Science, Gaziosmanpasa University, Tokat, Turkey
}

Received: 10.04.2020 \& Accepted: 12.05.2020

Doi: $10.12693 /$ APhysPolA.138.554

*e-mail: salih.saygi@gop.edu.tr

\begin{abstract}
An experimental study has been carried out for the velocity field characteristics of AlGaN on $\mathrm{AlN}$ /sapphire templates. A pulsed voltage input combination with a four-point measurement technique has been used to determine the drift velocity of electrons as a function of the applied field. Comparative data with earlier templates shows that a device performance depends on low-field mobility and saturated drift velocity.
\end{abstract}

topics: HEMT, drift velocity, AlGaN

\section{Introduction}

High electron mobility transistors, HEMT, fabricated from $\mathrm{AlGaN} / \mathrm{GaN}$ heterostructures are good nominees for microwave power devices. In these heterostructures, the polarization fields occur differently between the upper layer and the lower layer. The strain from the shape of the structure in one or both layers enhances an added built-in field due to the piezoelectric effect. The discontinuity in the normal electric field at the heterointerface produces a significant sheet carrier concentration crossing the $\mathrm{AlGaN}$ side of the interface. In addition, this discontinuity in the electric field can lead to higher carrier densities than in other conventional devices.

Thus far, high velocity and high thermal conductivity have been expected from high power semiconductor devices. The AlGaN/GaN structure attributes middling values of carrier heating to high velocity and high thermal conductivity. To perceive the expected characteristic values, the measurements of the velocity-field characteristics on these structures have to be given by comparative studies. However, each of the published reports apparently has not admitted the drift velocity for this heterostructure. In this work, we provide an accurate experimental setup and characteristic values for multilayer devices in order to optimize their design.

\section{Heterostructure material}

The device epilayer structure has been grown with a $3 \mu \mathrm{m}$ thick AlN buffer layer over basal plane sapphire substrates, using a growth procedure reported in [1]. The channel AlGaN layer was undoped and $0.6 \mu \mathrm{m}$ thick. The aluminum alloy composition and the thickness of the barrier layer for this study were at $85 \%$ and $270 \AA$, respectively. The gated and ungated multilayer structures with identical geometry intended for pulse measurement have formed on the undoped barrier samples using the standard photolithography and liftoff processing. After the mesa-reactive ion etching, $\mathrm{Zr} / \mathrm{Al} / \mathrm{Mo} / \mathrm{Au}$ contacts have deposited and annealed at the related temperature. To complete the wafer pattern, subsequently, $\mathrm{Ni} / \mathrm{Au}$ gates were formed.

\section{Setup and experimental procedure}

Figure 1 shows the epilayer structure and device geometry. The abbreviation used for the device is $\mathrm{Al}_{x} \mathrm{Ga}_{1-x} \mathrm{~N} / \mathrm{Al}_{y} \mathrm{Ga}_{1-y} \mathrm{~N} / \mathrm{AlN} / \mathrm{GaN} /$ Sapphire. To confirm that the device fabrication is acceptable for the measurements, TLM sheet resistances of the fabricated structure are well matched so that they correspond well with the value measured by the current measurement techniques.

All measuring devices are tested in the wafer form by probing. The experimental setup is shown in Fig. 2. For the measurement, a four-point measurement system was organized in the lab.

\begin{tabular}{|l|l|}
\hline S & D \\
\hline & Alo.8sGao.1sN \\
\hline & Alo.6sGao.3sN \\
\hline AlN Buffer \\
\hline Sapphire \\
\hline
\end{tabular}

Fig. 1. Epilayer structure and the device geometry. 




Fig. 2. Experimental setup.

The pulse setup is the first experimental prediction for this type of structure. In [2], Barker et al. have used a similar technique to measure typical bulk GaN test structures. To measure the high field transport properties of the 2DEG in the AlGaN/GaN heterostructure, the sample has been subjected to a pulsed voltage input. Due to the intrinsic nature of the pulse measurements, the voltage has been applied from outer probes to prevent transient heating. After monitoring the voltage dropping across the probe, one can calculate the current through the device. To prevent the high current failure on the device, the highresistance environment is used to monitor the outputs for measurements, e.g. an oscilloscope monitoring. The measurement has also been constructed for elevated temperatures. The temperature's increase and decrease have been observed by a thermocouple.

In [3-5], von Müench et al. have developed the same conductance technique for the experiment. Using the measured voltage and current, the drift velocity can be obtained with $v=I /(2 q w n t)$. The current $I$ is the current flowing through a region with the width $w$ and thickness $t$ while $n$ is the electron density, and $q$ is the electronic charge. As it can be observed, an accurate determination of the drift velocity involves a precise knowledge of the device dimensions. Therefore, the measurement exactly depends on how the device, both gated and ungated, is assembled. The electric field is calculated from the voltage drop across the closest contacts, and the resistance between the closest contacts. In an ideal case, the cross-section and physical composition are uniform within the sample, and the electric field and current density are both parallel and constant everywhere. The resistance for an ideal case material is expressed as $R=\rho t / A$. The device in our construction has no uniform pattern, hence the resistivity depends on structure. Furthermore, the current shows an anisotropic flow, therefore the device shape becomes significant. For our assembling device, the resistance between the closest contacts with a shape-related correction has been expressed as

$$
R_{\mathrm{end}}=\frac{\rho}{t} \log \left(I+\frac{s}{w}\right),
$$

where $\rho$ is the resistivity of the epilayer, $t$ is the thickness, $s$ the spacing between the devices, and $w$ is the width of the device. We believe that the expression for resistance in [4] also has meaningless unity (after unit analysis), but it was given with some correction to the measuring device. In the measuring device, the channel 3 counts up the resistance with respect to the inner resistance.

\section{Discussion of the outcomes}

The DC pulse measurement has been carried out after configuring the experiment in the lab. Figure 3 shows the comparison of the drift velocity outcomes obtained for several geometries. The graph depicts the data associated with experiments and calculations given by references. This work and the data of Ardaravičius et al. [6] show the experimental result which clarifies the similarity with the calculated data. Our electron drift velocity data has been fitted with the empirical value of drift velocity

$$
v=\frac{\mu E}{\left[1+\left(\frac{\mu E}{v_{s}}\right)^{\sigma}\right]^{1 / \sigma}} .
$$

In the fitting equation (2), $v$ is the electron velocity, $E$ is the electric field, $\mu$ is the mobility, $v_{s}$ is the electron saturation velocity, and $\sigma$ is a dimensionless fitting parameter. Preliminary observations of different data are comparable to each other. The highest drift velocity field achieved in the dataset is $\approx 150 \mathrm{kV} / \mathrm{cm}$. A sharp current transient can be observed above this value.

The drift velocities given experimentally are lower than the predicted simulation values. The explanation is twofold. Firstly, the electron drift velocity measured with a particular substrate device is considerably lower than that of the device previously reported with a GaN material. Secondly, the maximum electron drift velocity decreases after a limiting value of the electric field. Our data also confirms this implication. We assumed that the electric field is enhanced near the drain and reduced near the edge of the source due to variation in density. The inhomogeneity can also be explained by the fact that the discontinuity between the two sides of the heterojunction occur by the carrier layer. For the inhomogeneity, Kachorovskii et al. [7] have suggested some kinetic instability through the sample for heterojunction. In other words, if the current flows through the carrier layer at high fields where the velocity field curve is non-linear, the instabilities can be caused by the electrostatics of this field discontinuity. Thus, the current conservation has always been maintained [8].

Another important observation based on the graph is that after an electric field limiting value, the drift velocity values have been distributed without the loss of generality. Unfortunately, we are unable to decide whether the point is a high electric field limiting point or not. Less than $150 \mathrm{kV} / \mathrm{cm}$ in electric field, the drift velocity 


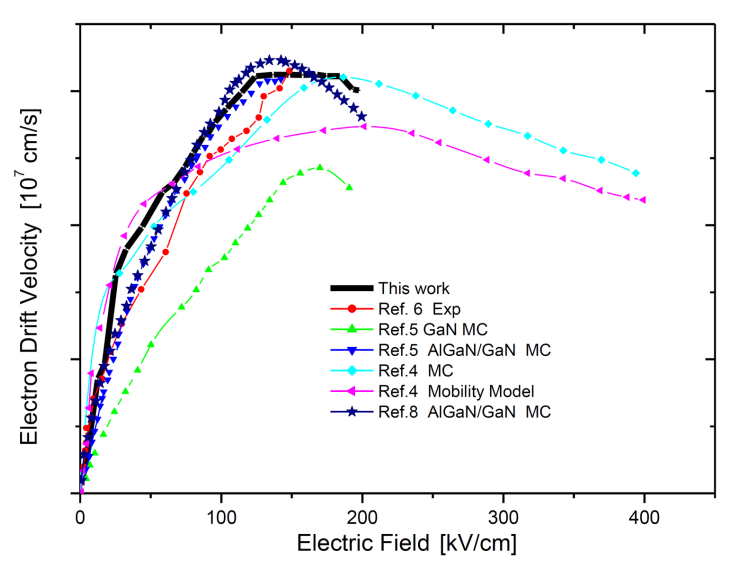

Fig. 3. The experimental and calculated drift velocities for some constricted geometries.

shows a linear behavior for all the devices and also for the references. Remarkably, all data at the electric field, $\sim 80 \mathrm{kV} / \mathrm{cm}$, show the same value of drift velocity: $\sim 2.5 \times 10^{-7} \mathrm{~cm} / \mathrm{s}$. If $80 \mathrm{kV} / \mathrm{cm}$ is a limiting value of the electric field, it needs to be explained clearly.

\section{Results}

In combination with theoretical efforts, we have attempted the experimental measurement of electron velocity as a function of the electric field on an advanced AlGaN/GaN device. We have based our conclusions on comparison. First, the peak electron velocity remains the same for all data values, second, the electron drift velocity of the AlGaN/GaN device is significantly lower than in the previously reported devices of the GaN material in which there is no heterostructure constructed. Third, the maximum electron drift velocity decreases after a limiting value of the electric field. Fourth, all data in a region with a weak electric field (where it is less than the limit value) show a linear behavior on the drift velocity, as it has been be observed. Last, after a limiting value of the electric field, the velocity values are scattered without losing generality.

\section{References}

[1] F. Qhalid, V. Adivarahan, G. Mikhail, K. Thomas, M. Jin, A. Fernando, I. Ponce, A. Khan, Jpn. J. Appl. Phys. 46, 29 (2007).

[2] J.M. Barker, D.K. Ferry, S.M. Goodnick, D.D. Koleske, A.E. Wickenden, R.L. Henry, Microelectron. Eng. 63, 193 (2002).

[3] W.V. Muench, E. Pettenpaul, J. Appl. Phys. 48, 4823 (1977).

[4] I.A. Khan, J.A. Cooper, IEEE Trans. Electron. Devices 47, 269 (2000).

[5] J.M. Barker, R. Akis, T.J. Thornton, D.K. Ferry, S.M. Goodnick, Phys. Status Solidi Appl. Mater. Sci. 190, 263 (2002).

[6] L. Ardaravičius et al., Semicond. Sci. Technol. 30, 10 (2015).

[7] Y. Kachorovskii, S. Lyubinskiy, D. Tsendin, Phys. Rev. B 68, 1 (2003).

[8] T.H. Yu, K.F. Brennan, J. Appl. Phys. 91, 3730 (2002). 